Inventory of Glaciers, Glacial Lakes and Glacial Lake Outburst Floods

Monitoring and Early Warning Systems in the Hindu Kush.Himalayan Region

Bhutan

Pradeep KK. Mool Dorji Wangda Samjwal R. Bajpacharya Karma Kunzang Deo R. Gurung Sharad P. Joshif 



\section{Inventory of Glaciers, Glacial Lakes and Glacial Lake Outburst Floods Monitoring and Early Warning Systems in the Hindu Kush-Himalayan Region Bhutan}

Pradeep K. Mool, Dorji Wangda, Samjwal R. Bajracharya, Karma Kunzang, Deo Raj Gurung, Sharad P.Joshi 
Copyright (C) 2001

International Centre for Integrated Mountain Development

All rights reserved

\section{Cover plate:}

Front: Three-dimensional perspective computer-generated view of Raphstreng Tsho showing the Thorthomi glacier and Lugge Tsho area, and the effect of the Lugge Tsho GLOF in the Pho Chu area (1999 IRS1D LISS3 and PAN data)

\section{Back plates:}

The Pho Chu basin area in Bhutan which includes the Lugge Tsho, Raphstreng Tsho, and their associated glaciers

Top: $\quad$ Lugge Tsho Glacial Lake two weeks after the GLOF of 7 October 1994

$$
\text { - Yeshi Dorji }
$$

\section{Bottom clockwise:}

Satellite image (IRS1C PAN) of 3 January 1999 draped on a digital elevation model (DEM) derived from topographic maps showing the breached area of Lugge Tsho Lake Field photo of Thorthormi Glacier and Lugge Tsho showing the breach point of Lugge Tsho - Phuntso Norbu

Satellite image (IRS1D PAN) of 3 December 2000 showing Punakha Dzong, the impact area of the GLOF

Field photo of Punakha Dzong three days after the disaster of 1994

- Phuntso Norbu

\section{Published by}

International Centre for Integrated Mountain Development

G.P.O. Box 3226

Kathmandu, Nepal

ISBN929115 3621 (For CD-ROM Version)

\section{Editorial Team}

Greta Mary Rana (Senior Editor)

Diana Gallannaugh, Consultant Editor

Dharma R. Maharjan (Technical Support and Layout)

\section{Printed and bound in Nepal by}

Quality Printers Pvt. Ltd

Kathmandu 



\section{Foreword}

The glaciers of the Hindu Kush-Himalayas $(\mathrm{HKH})$ are nature's renewable storehouse of fresh water from which hundreds of millions of people downstream benefit just when it is most needed - in the dry hot season before the monsoons. While the total number of glaciers in the region is still unknown, this study has for the first time documented that there are 677 glaciers in Bhutan alone. Covering an area of 1,317 square kilometres, these high frozen reservoirs release their water at the top of their watersheds. They serve as the perennial sources of rivers that wind their way through grazing, agricultural, and forest lands and are used as renewable sources of irrigation, drinking water, energy, and industry.

However, these glaciers are retreating in the face of accelerating global warming. They are particularly vulnerable to climate change, and the resultant long-term loss of natural fresh water storage will have as yet uncalculated effects on communities downstream. More immediately, as glaciers retreat, glacial lakes form behind some of the now exposed terminal moraines. Rapid accumulation of water in glacial lakes, particularly in those adjacent to receding glaciers, can lead to a sudden breaching of the unstable 'dam' behind which they have formed. The resultant discharges of huge amounts of water and debris - a glacial lake outburst flood or GLOF - often have catastrophic effects downstream.

Many glacial lakes are known to have formed in the $\mathrm{HKH}$ in the last half century and a number of GLOFs have been reported in the region, including in Bhutan, in the last few decades. These GLOFs have resulted in many deaths, as well as the destruction of houses, bridges, fields, forests and roads. The lakes at risk, however, are situated in remote and inaccessible areas. When they burst, the local communities may have been devastated, while those in far away cities were largely unaware of the event.

In Bhutan, the catastrophic Lugge Tsho glacial lake flood in 1994, which followed a similar event in nearby Nepal in 1985, raised awareness of the problem considerably. As described in this publication, a partial burst from this lake caused loss of life and property along the PunakhaWangdue valley, damaged part of the Dzongchung of Punakha Dzong, and washed away or covered nearly 1,000 acres of pastureland.

Despite numerous studies of individual cases, there is still no detailed inventory of glaciers and glacial lakes, of GLOF events or of potential GLOF sites, in the HKH region - let alone of their impact on downstream populations and investments. This publication, along with the sister publication on the glaciers and glacial lakes of Nepal, is designed to begin filling this pressing need. The research upon which it is based started in 1999, when the United Nations Environment Programme Regional Resource Centre for Asia and the Pacific (UNEP/RRC-AP) provided ICIMOD with the opportunity of using its expertise in the area of geographic information systems (GIS) to create a comprehensive inventory and GIS database of glaciers and glacial lakes in Nepal and Bhutan using available maps, satellite images, aerial photographs, reports, and field data on different scales. It built on ICIMOD's experience and long-standing concern with collecting and distributing material on the means to identify and mitigate mountain disasters and safeguard the livelihoods of vulnerable mountain people and their downstream neighbours.

One of the study's major objectives was to identify areas where GLOF events had occurred and lakes that could pose a potential threat of a GLOF in the near future. Out of a surprisingly large total of 2,674 glacial lakes, the researchers found 24 lakes that are potentially dangerous. These results thus provide the basis for development of a monitoring and early warning system and for the planning and prioritisation of disaster mitigation efforts that could save many lives and properties situated 
downstream, as well as guide infrastructure planning. In addition, it is anticipated that this study will provide useful information for many of those concerned with water resources and land-use planning.

As a presentation of the first results of the UNEP/RRC-AP supported study, this publication also includes a description of the methods used to identify glaciers, glacial lakes, and glacial lakes that may pose a threat; as well as an inventory (and maps) of the glaciers and glacial lakes in Bhutan. It includes a summary of the results of studies of various glacial lakes, and a brief review of the causes and effects of known GLOF events in Bhutan. The database and analysis are the first to cover the whole of the country on a large scale.

We are thus confident that this comprehensive report and digital database will be of service to scientists, planners, and decision-makers in many areas. Through their informed actions, we hope it will contribute to improving the lives of those living in the mountains, and help safeguard future investments for the benefit of many people in the region.

ICIMOD is grateful to UNEP/RRC-AP for its support to this work and the strong support and advice given while carrying out the project. We are also pleased that this project has enabled us to continue to strengthen our collaboration with the Department of Geology and Mines of the Royal Government of Bhutan and to continue to assist in developing regional capacity and co-operation.

J Gabriel Campbell

Director General ICIMOD 
We express our sincere thanks to the Department of Geology and Mines of the Royal Government of Bhutan, which has been closely affiliated with the present study and made available important information on maps, satellite images, reports and data. We thank Dr. Hari Man Shrestha, the former executive secretary of WECS, who carried out a technical review of the study and Dr. V. Galay and Dr. M. Zimmerman for their valuable comments and suggestions on the draft report. Mr. Basanta Shrestha, Acting Head, MENRIS, coordinated and made valuable inputs to the study. Other ICIMOD staff members who have assisted in the study include Ms. Monica Moktan, Ms. Rajani Bajracharya, Prof. Li Tianchi, Mr. Sushil Pradhan, Mr. Birendra Bajracharya, Mr. Sushil Pandey, Mr. Saisab Pradhan, Mr. Anirudra M. Shrestha, and Mr. Govinda Joshi. We would like to thank them all for their contributions. We would also like to thank Mr. Dharma Ratna Maharjan for the layout design and Mr. Asha Kaji Thaku for cartographic work. Mr. Pramod Pradhan, former Head of the MENRIS division of ICIMOD, is thanked for his valuable inputs and for coordinating the project process. Last but not least we wish to thank Mr. Surendra Shrestha, Regional Coordinator, Mr. Dola Govinda Pradhan, Dr. Chandra Giri, Mr. Mylvakanam Iyngararasan, Ms. May Ann E. Mamicpic, and Ms. Kritiya Gajesani of UNEP/RRC-AP for their timely and strong support and advice while implementing the project. 



\section{Editor's note}

The terms glacier lake and glacial lake are often used interchangeably to refer to any lake associated with a glacier, regardless of the means of formation, although some investigators restrict their definitions to particular types of lake. For the purposes of this document, all lakes in contact with or near a glacier, or formed by recent glacial morphology, are referred to as 'glacial lakes'. In practice, most are of the type produced on a glacier's perimeter by meltwater from the glacier, by many termed a 'proglacial lake'.

The terms 'Himalaya' and 'Himalayas' are use to refer to the geological formation and the geographical region, respectively. 



\section{Abbreviations and Acronyms}

CD

compact disk

DEM digital elevation model

DGM Department of Geology and Mines

DOR Department of Roads

EAP-AP Environment Assessment Programme - Asia Pacific

EMS electromagnetic spectrum

ERTS Earth Resources Technology Satellite

ESCAP Economic and Social Commission for Asia and Pacific

ETH Swiss Federal Institute of Technology

FCC false colour composite

GDP gross domestic product

$\mathrm{Gl}$ glacial lake

GIS geographic information system

GLOF glacial lake outburst flood

$\mathrm{Gr} \quad$ glacier

GSB Geological Survey of Bhutan

GSI Geological Survey of India

GTZ Deutsche Gesellschaft für Technische Zusammenarbeit

(German Agency for Technical Cooperation)

$\mathrm{HKH} \quad$ Hindu Kush-Himalayas

HRV High Resolution Visible sensor (SPOT)

ICIMOD International Centre for Integrated Mountain Development

ILWIS Integrated Land and Water Information Systems

IR Infrared

IRS1C Indian Remote Sensing Satellite series 1C

IRS1D Indian Remote Sensing Satellite series 1D

Landsat Land Resources Satellite

LIGG Lanzhou Institute of Glaciology and Geocryology

LISS Linear Imaging and Self Scanning sensor

masl metres above sea level

MBT Main Boundary Thrust

MCC Meteor Communication Corporation

MCT Main Central Thrust

MENRIS Mountain Environment and Natural Resources' Information System

MOS Marine Observation Satellite

MSS Multi Spectral Scanner (Landsat)

NEA Nepal Electricity Authority

NRSA National Remote Sensing Agency

PAN Panchromatic Mode sensor system (SPOT) 

Royal Bhutan Army

RGOB

RS red green blue

SPOT Royal Government of Bhutan remote sensing

SWIR

Système Probatoire Pour l'Observation de la Terre / Satellite Pour l'Observation de la Terre

SWIR Short Wave Infra Red sensor

TM Thematic Mapper (Landsat)

TTS Temporary Technical Secretary

UNDP United Nations Development Project

UNEP United Nations Environment Programme

VNIR Visible and Near Infra Red (AVNIR)

WAPCOS Water and Power Consultancy Services (India) Limited

WECS

WGI

Water and Energy Commission Secretariat

WGMS

WWW

World Glacier Inventory

World Glacier Monitoring Service

World Wide Web

XS

Multispectral Mode sensor system (SPOT) 


\section{Contents}

Foreword

Acknowledgements

Editor's note

Abbreviations and Acronyms

Chapter 1 - Introduction to Inventory of Glaciers and Glacial Lakes.......................................... 1

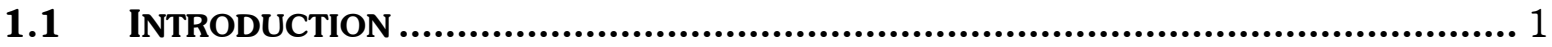

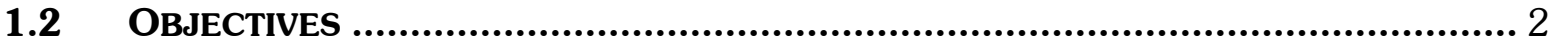

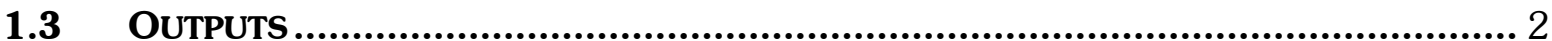

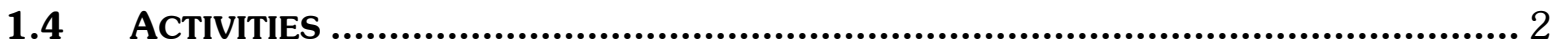

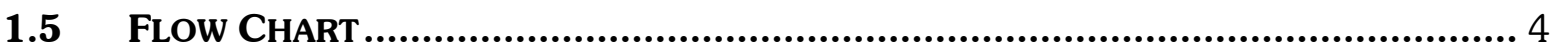

Chapter 2 -General Characteristics of the Country .................................................................. 5

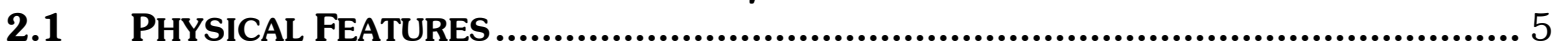

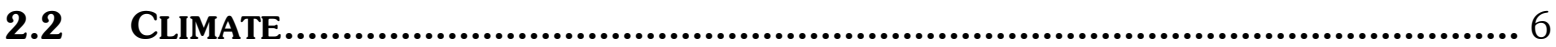

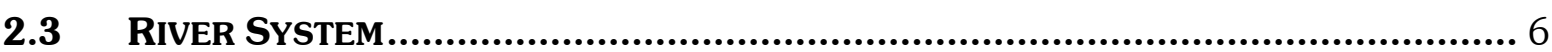

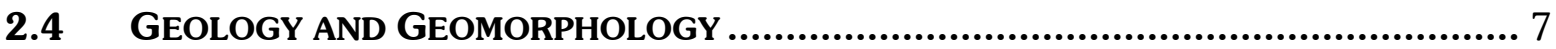

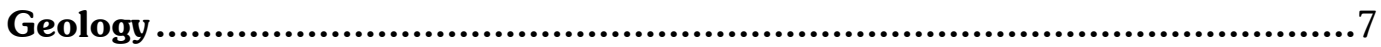

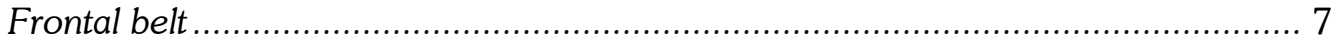

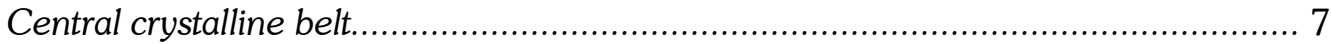

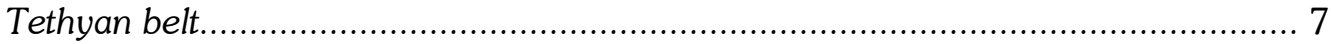

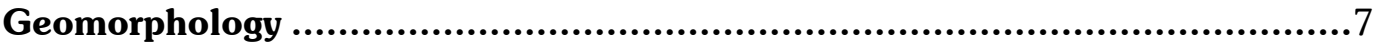

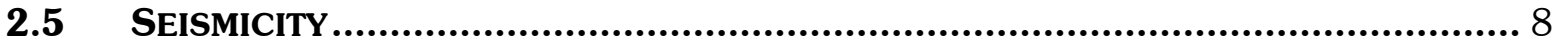

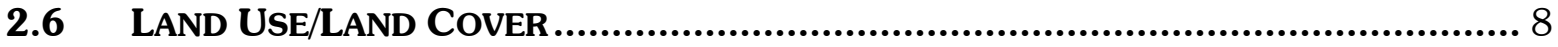

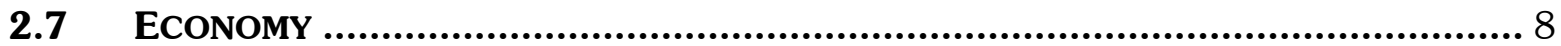

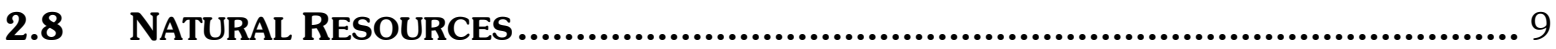

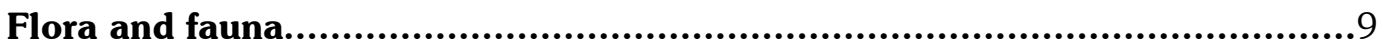

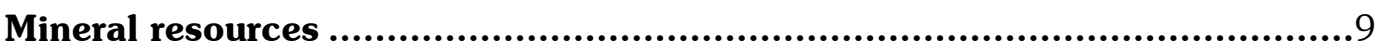

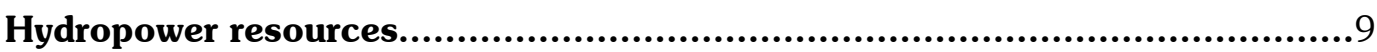

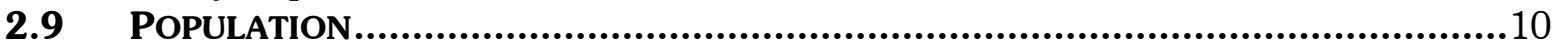

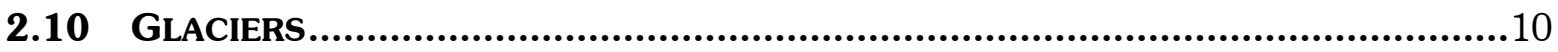

2.11 GLACIAL LAKES ........................................................................................11

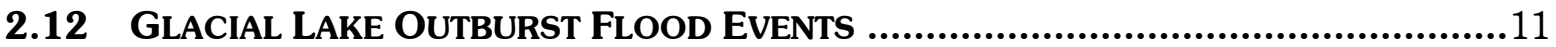

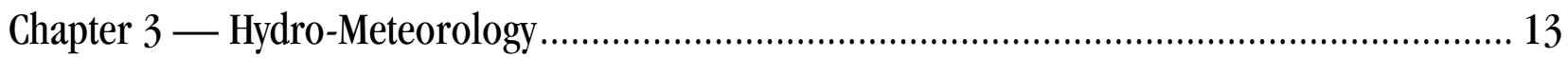

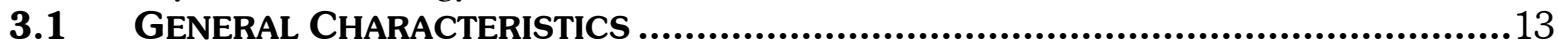

The Amo Chu basin ................................................................................... 13

The Wang Chu basin ................................................................................... 13

The Puna Tshang Chu basin (Sankosh) ........................................................ 14

The Manas River basin ........................................................................... 14

The Nyere Ama Chu basin..................................................................... 15

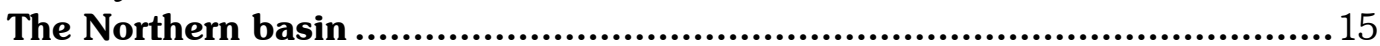

3.2 HYDRO-METEOROLOGICAL OBSERVATION ......................................................16

Meteorological observation ....................................................................16

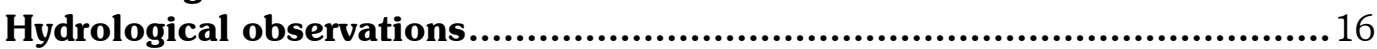

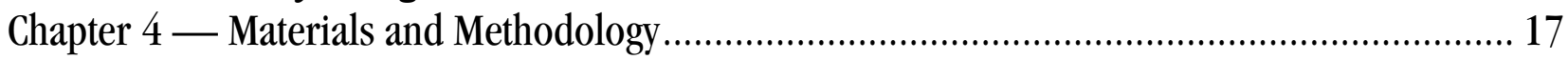




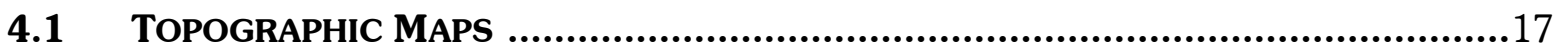

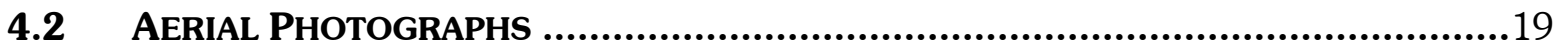

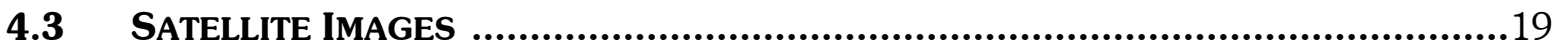

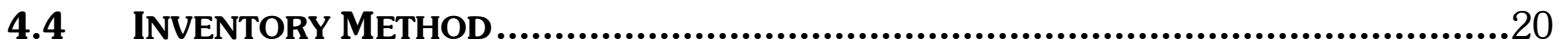

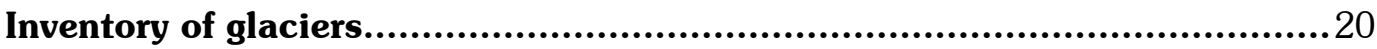

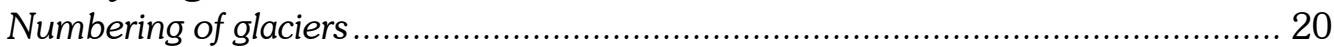

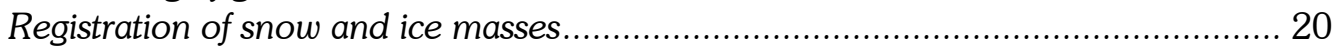

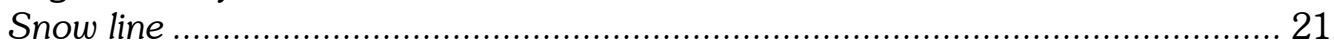

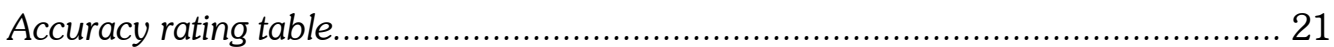

Mean glacier thickness and ice reserves............................................................ 21

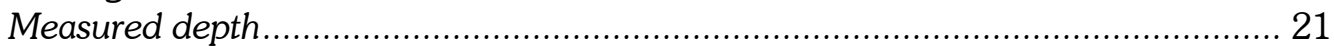

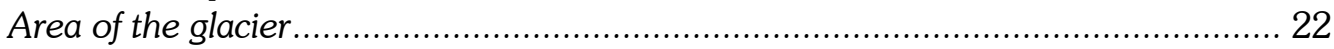

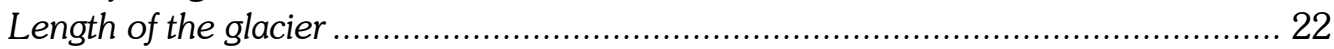

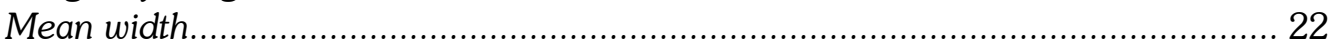

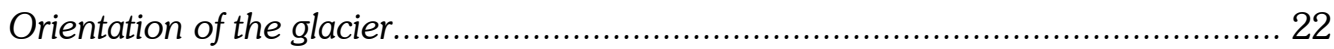

Elevation of the glacier..................................................................................... 22

Morphological classification …………..........................................................22

Inventory of glacial lakes ....................................................................26

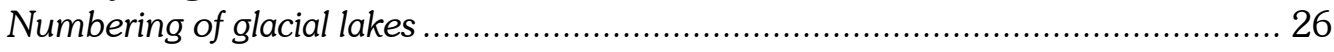

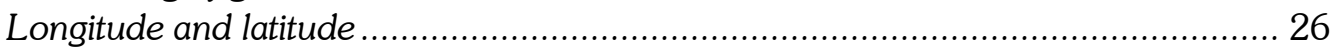

Area.

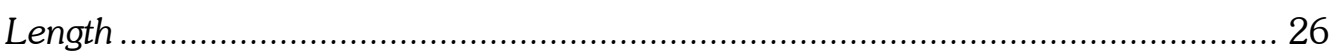

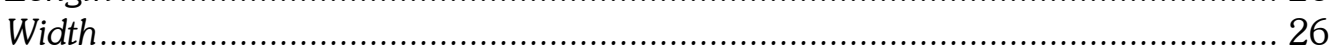

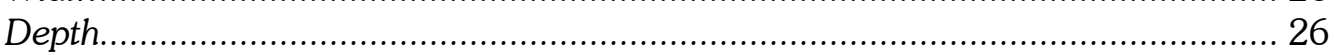

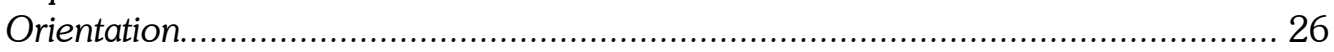

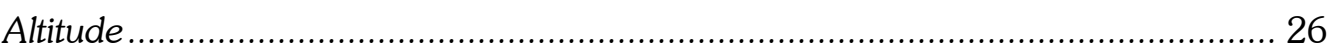

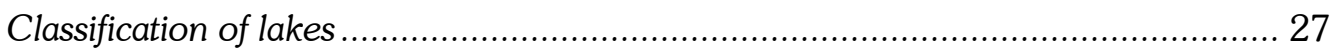

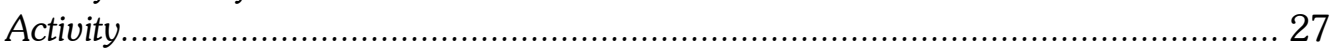

Types of water drainage ............................................................................... 27

Chemical properties...................................................................................... 27

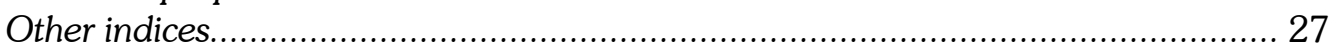

Chapter 5 - Spatial Data Input and Attribute Data Handling.................................................... 29

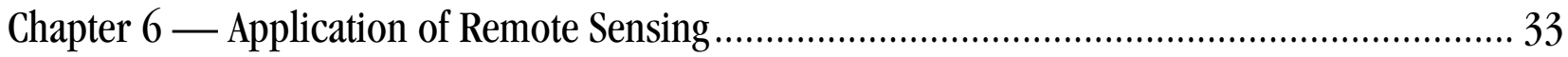

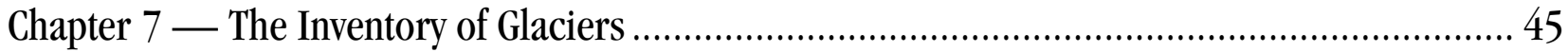

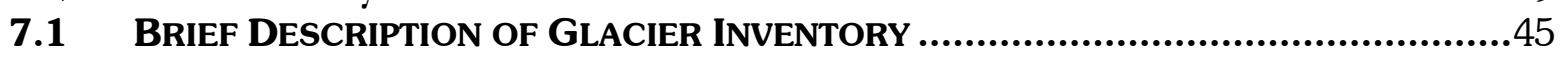

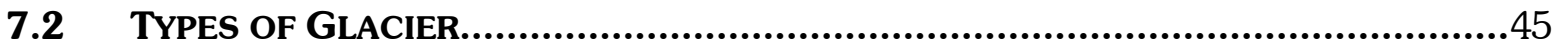

7.3 GENERAL CHARACTERISTICS OF GLACIATION ..................................................

7.4 GLACIERS OF BHUTAN..............................................................................46

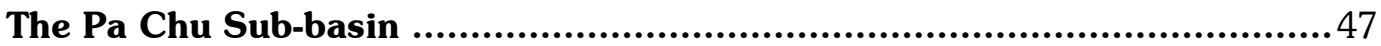

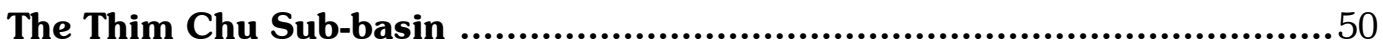

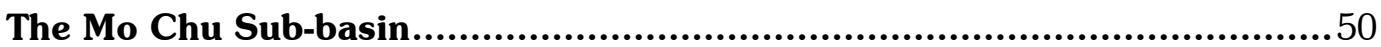

The Pho Chu Sub-basin............................................................................52

The Mangde Chu Sub-basin.......................................................................5

The Chamkhar Chu Sub-basin..................................................................5

The Kuri Chu Sub-basin .........................................................................5

The Dangme Chu Sub-basin ....................................................................60

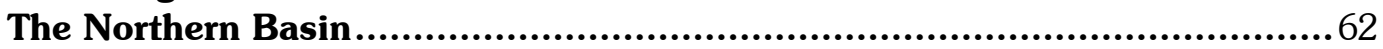

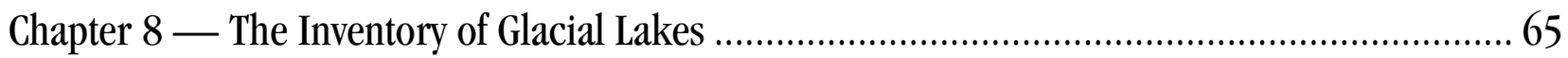

8.1 BRIEF DESCRIPTION OF GLACIAL LAKE INVENTORY .........................................65

8.2 GlaCial LAKES-THEIR NUMBERING, TYPE, AND ChARACTERISTICS ................65 


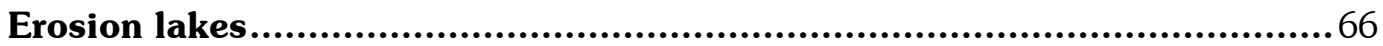

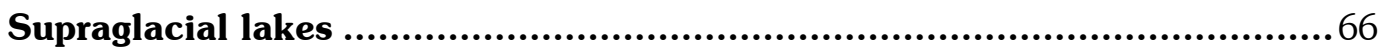

Moraine-dammed lakes .................................................................66

Ice-dammed lakes........................................................................6. 67

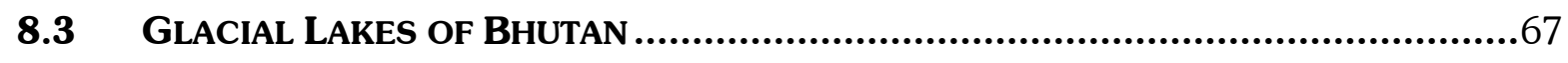

The Amo Chu basin ...................................................................6 67

The Wang Chu basin ................................................................6 67

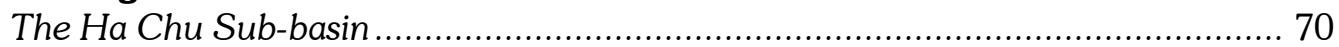

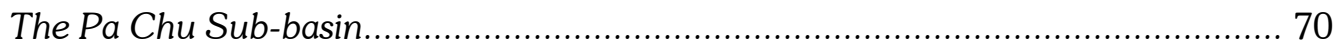

The Thim Chu Sub-basin.............................................................................. 70

The Puna Tshang Chu (Sankosh) basin ........................................... 72

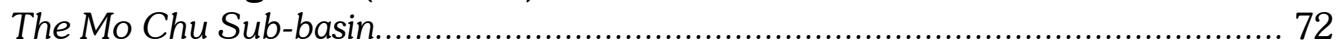

The Pho Chu Sub-basin................................................................................. 72

The Dang Chu Sub-basin................................................................................... 75

The Manas Chu basin................................................................. 76

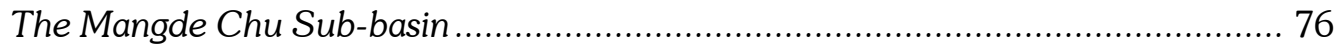

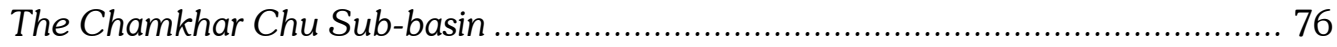

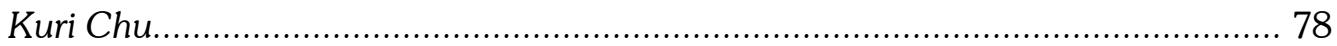

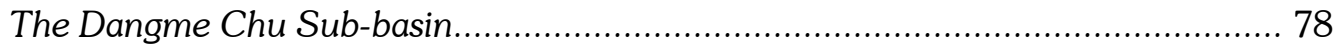

The Nyere Ama Chu basin............................................................. 78

The Northern basin .................................................................. 80

Chapter 9 — Glacial Lake Outburst Floods and Damage in the Country................................. 81

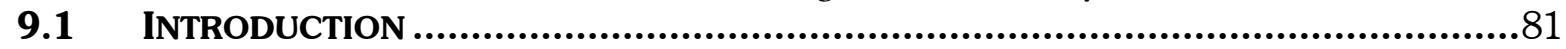

9.2 CAUSES OF LAKE CREATION ................................................................81

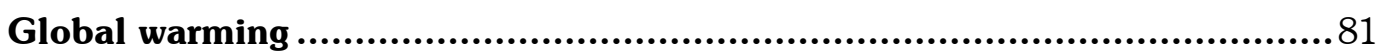

Glacier retreat ........................................................................ 81

Causes of glacial lake water level rise .............................................. 82

9.3 BURSTING MECHANISMS ......................................................................8

Mechanism of ice core-dammed lake failure ..................................... 82

Mechanism of moraine-dammed lake failure...................................... 83

9.4 SURGE PROPAGATION ..................................................................... 84

9.5 SEDIMENT PROCESSES DURING A GLACIAL LAKE OUTBURST FLOOD..................86

9.6 SOCIOECONOMIC EFFECTS OF GLACIAL LAKE OUTBURST FLOODS....................86

9.7 BRIEF REVIEW OF Glacial LAKE OUTBURST FlOOD EVENTS AND DAMAGE

CAUSED .....................................................................................8

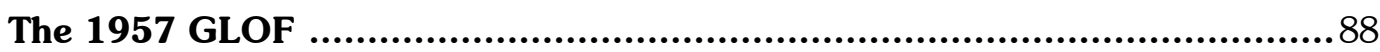

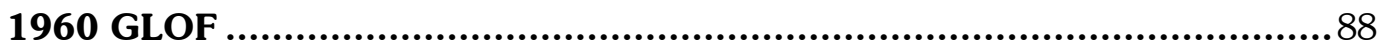

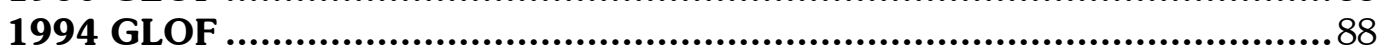

Chapter 10 — The Glacial Lakes Studied in Bhutan.......................................................... 95

10.1 Previous STUdIES ......................................................................95

Lunana lake expedition (July to September 1986) ................................ 95

Joint expedition team (1995) .......................................................... 96

Expedition to Roduphu glacial lake (1996) .......................................... 96

Japan-Bhutan joint research programme (1998) ................................ 97

10.2 GlaCIAL LAKES STUDIED .............................................................97

Raphstreng Tsho (eastern Lunana).................................................. 97

Hydrometry of the lake outlet stream ............................................................ 98

Suspended sediment transport studies of the lake and outlet stream...................... 98

Ice flow studies of the glacier feeding the lake.................................................... 98

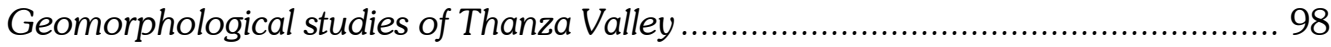

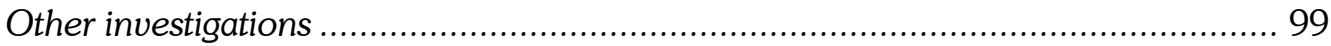




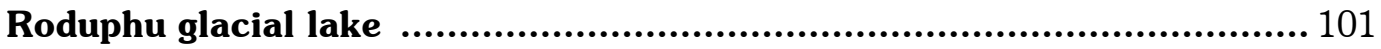

Tshokar Tsho glacial lake ............................................................... 101

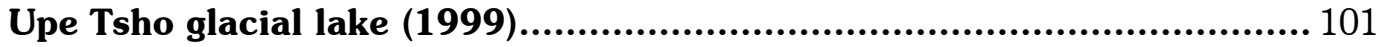

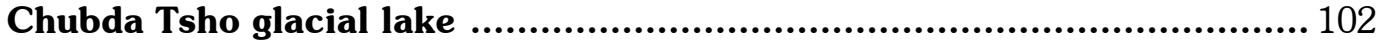

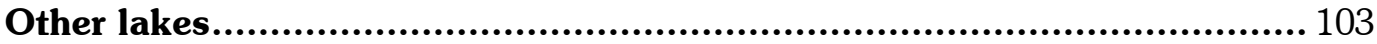

Chapter 11 — The Potentially Dangerous Glacial Lakes .......................................................... 105

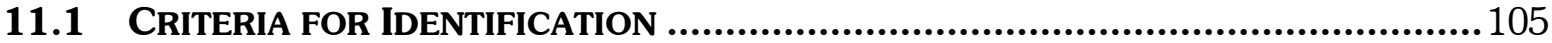

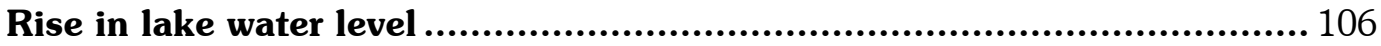

Activity of supraglacial lakes ......................................................... 106

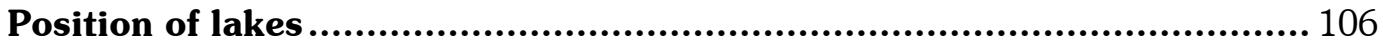

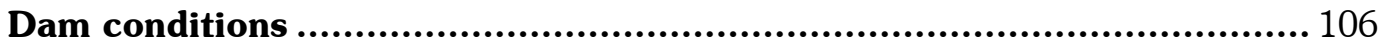

Condition of associated mother glacier ............................................. 107

Physical conditions of surroundings .................................................. 107

11.2 POTENTIALLY DANGEROUS GlaCIAL LAKES......................................... 107

Chapter 12 - Glacial Lake Outburst Flood Mitigation Measures, Monitoring and Early

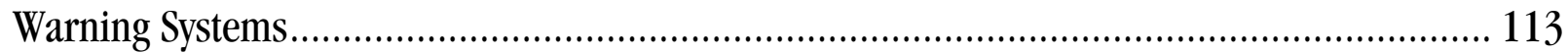

12.1 REDUCING THE VOLUME OF LAKE WATER .................................................113

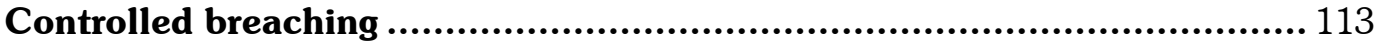

Construction of an outlet control structure .......................................... 114

Pumping or siphoning out the water from the lake ................................. 114

Making a tunnel through the moraine dam.......................................... 114

12.2 Preventative Measures Around the LaKe AREA..........................................114

12.3 Protecting Infrastructure Against the Destructive Forces of THE

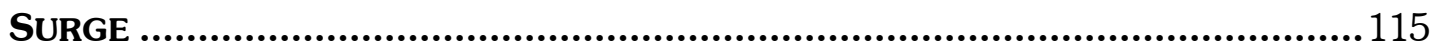

12.4 MONITORING AND EARLY WARNING SYSTEMS ..............................................115

12.5 Mitigation MEASURES, MONITORING, AND EARLY WARNING SYSTEMS APPLIED

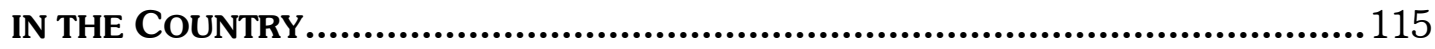

Flood mitigation measures (Phase I-1996).................................... 116

Raphstreng Tsho outburst flood mitigation project (Lunana)

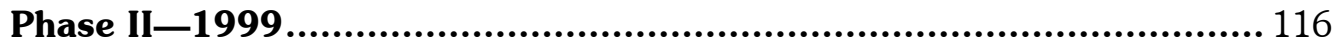

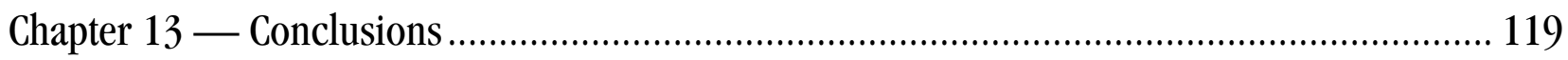

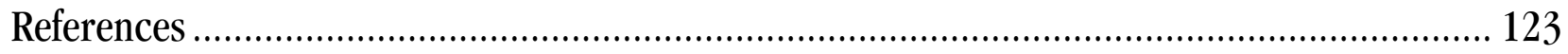

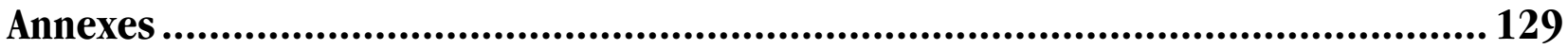

